\title{
COMPARAÇÃO FLORÍSTICA E FITOSSOCIOLÓGICA ENTRE COMUNIDADES DE PALMEIRAS DE FLORESTAS SECUNDÁRIAS E PRIMÁRIA DA ÁREA DE PROTEÇÃO AMBIENTAL 'IGARAPÉ SÃO FRANCISCO', ACRE, BRASIL
}

\author{
Simony Hechenberger ${ }^{1}$, Evandro José Linhares Ferreira ${ }^{2}$ \\ ${ }^{1}$ Engenheira Florestal, Mestranda em Ciências Florestais, Universidade Federal do \\ Acre, Rio Branco, Acre (simony.ac@gmail.com). \\ 2 Doutor em Botânica, Pesquisador do Instituto Nacional de Pesquisas da Amazônia \\ - INPA, Rio Branco, Acre.
}

Recebido em: 08/04/2017 - Aprovado em: 10/06/2017 - Publicado em: 20/06/2017 DOI: 10.18677/EnciBio 2017A170

O desmatamento ocorrido na Amazônia nestes últimos 30 anos tem resultado na fragmentação das florestas da região. A fragmentação pode alterar a diversidade e a composição florística dos fragmentos formados e processos ecológicos como a ciclagem de nutrientes, polinização e a capacidade de estocar carbono. Na Amazônia as palmeiras são importantes componentes da vegetação e muitas espécies são chaves como recurso alimentar da fauna silvestre. Assim, a diminuição na densidade de palmeiras poderá causar mudanças imprevisíveis na estrutura das florestas e na cadeia alimentar. Nesse contexto, este estudo objetivou conhecer e comparar a composição florística e alguns parâmetros fitossociológicos de comunidades de palmeiras em três fragmentos florestais secundários de diferentes idades e um fragmento primário na APA 'Igarapé São Francisco', em Rio Branco, Acre, para avaliar as alterações decorrentes da fragmentação e da antropização ocorridas. Os resultados obtidos indicam que a flora de palmeiras da APA é pobre quando comparada com a observada em outras florestas da região. Fragmentos mais antigos apresentam maior riqueza de gêneros e espécies, mas as comunidades de palmeiras encontradas são estruturalmente anormais, com ausência ou baixo número de plântulas. A espécie mais abundante foi Attalea phalerata, típica de florestas em estágios iniciais de regeneração, mas dominante no fragmento com 35-40 anos, sugerindo que as modificações paisagísticas na APA podem estar favorecendo essa espécie. No fragmento mais recente (5-10 anos), a sobrevivência da comunidade de palmeiras está comprometida por alterações antrópicas (incêndios florestais), pela fragmentação e a invasão do bambu (Guadua spp.) no sub-bosque da floresta.

PALAVRAS-CHAVE: Amazônia, Arecaceae, Fragmentação. 


\title{
FLORISTIC AND PHYTOSOCYOLOGICAL COMPARISON BETWEEN PALM COMMUNITIES FROM SECONDARY AND PRIMARY FORESTS OF THE ENVIRONMENTAL PROTECTION AREA 'IGARAPÉ SÃO FRANCISCO', ACRE, BRAZIL
}

\begin{abstract}
The Amazon deforestation in the last 30 years resulted in the fragmentation of the region's forests. Fragmentation can alter the diversity and floristic composition of the fragments formed and ecological processes such as nutrient cycling, pollination and the ability to store carbon. In the Amazon, palms are important components of vegetation and many species are key resources for the wildlife. Thus, a decrease in palm density may cause unpredictable changes in the forest structure and the food chain. This study aimed to know and compare the floristic composition and some phytosociological parameters of palm communities in three secondary forest fragments of different ages and a primary fragment in the APA 'Igarapé São Francisco', in Rio Branco, Acre, to evaluate the alterations due to the fragmentation and anthropization. The results indicate that the APA palm flora is poor when compared to that of other surrounding forests. The oldest fragments show a greater richness of genera and species, but the palm communities found are structurally abnormal, with the absence or low number of seedlings. The most abundant species was Attalea phalerata, typical of early regeneration forests, but dominant in the fragment with $35-40$ years, suggesting that the landscape modifications in the APA may be favoring this species. In the younger fragment (5-10 years), of the palm community survival is compromised by anthropic changes (forest fires), the fragmentation and invasion of bamboo (Guadua spp.) in the forest understory.
\end{abstract}

KEYWORDS: Fragmentation, Amazon, Arecaceae.

\section{INTRODUÇÃO}

desmatamento em curso por toda a região amazônica nos últimos 30 anos tem resultado na formação de mosaicos de diferentes formatos e tamanhos constituídos, em sua maioria, por fragmentos florestais primários ou secundários em diferentes estádios de regeneração, áreas de pastagem e cultivos agrícolas (MEDEIROS et al., 2013; ARIMA et al., 2016). Esta fragmentação da floresta pode ter múltiplos efeitos para as comunidades florestais impactadas, com destaque para alterações na diversidade e na composição florística dos fragmentos formados (ARROYO-RODRÍGUEZ et al., 2013), e em processos ecológicos como a ciclagem de nutrientes, polinização e a capacidade da floresta de estocar carbono (TABARELLI et al., 2012; PUTZ et al., 2014). Muitas dessas alterações estão relacionadas com o tamanho da área fragmentada, a extensão de sua borda, distância entre os fragmentos e a composição original do habitat matriz (LAURANCE \& VASCONCELOS, 2009).

Em geral, as alterações microclimáticas e ecológicas que ocorrem nas áreas florestais fragmentadas causam, em longo prazo, uma deterioração da biodiversidade local e afetam de maneira distinta as populações das diferentes espécies de plantas existentes nessas áreas (TILMAN et al., 1994; BENCHIMOL \& PERES, 2015). Isso pode resultar, entre outras consequências, em uma redução da densidade de indivíduos e de espécies que favorece a sobrevivência e o aumento das populações mais resistentes ao estresse ambiental, caso das espécies em início de sucessão, e provocar a diminuição ou mesmo a extinção de espécies mais suscetíveis, como as tardias (SANTOS-SILVA et al., 2013; MESQUITA et al., 2015). ENCICLOPÉDIA BIOSFERA, Centro Científico Conhecer - Goiânia, v.14 n.25; p. 345 
$\mathrm{Na}$ Amazônia as palmeiras são importantes componentes florísticos da maioria das tipologias florestais e muitas de suas espécies são consideradas chave para o fornecimento de recursos alimentares para a fauna silvestre (SCARIOT, 1999; ter STEEGE et al., 2013; SILVA et al., 2015;). Uma eventual diminuição na densidade da população de palmeiras decorrente da fragmentação das florestas da região poderá causar mudanças imprevisíveis na estrutura dessas florestas e na cadeia alimentar, tendo como consequência direta a diminuição do valor biológico dessas florestas (SCARIOT, 1999).

No Acre, CARVALHO et al. (2010) observaram que a fragmentação ocorrida na cobertura florestal da Área de Proteção Ambiental (APA) 'Raimundo Irineu Serra' alterou negativamente a composição florística e diminuiu a riqueza e a diversidade de muitas espécies de palmeiras, favorecendo a dominância nos fragmentos remanescentes de uma ou poucas espécies.

Para conhecer o comportamento e a dinâmica das comunidades vegetais de uma determinada área é necessário identificar as espécies presentes, determinar sua composição florística e realizar estudos fitossociológicos, especialmente os relacionados com a sua estrutura horizontal, para determinar parâmetros - como densidade, dominância, frequência e importância das espécies - que possam ajudar na compreensão da dinâmica de vida das espécies integrantes dessas comunidades (CHAVES et al., 2013; ARAÚJO et al., 2015;). Essas informações são indispensáveis para planejar medidas de mitigação de impactos decorrentes das alterações naturais ou promovidas pelo homem e executar programas de recuperação, restauração e conservação das áreas afetadas (SILVA et al., 2013).

Nesse contexto, o presente estudo objetivou conhecer e comparar a composição florística e alguns parâmetros fitossociológicos de comunidades de palmeiras de três fragmentos florestais secundários em diferentes estádios de regeneração e um fragmento primário na APA 'Igarapé São Francisco', em Rio Branco, Acre, para avaliar as alterações que elas sofreram em razão da fragmentação e da antropização sofrida pelas florestas que elas integram.

\section{MATERIAL E MÉTODOS}

\section{Caracterização da área de estudo}

A APA Igarapé São Francisco localiza-se na região leste do Acre e integra a área dos municípios de Rio Branco e Bujari (Figura 1) (ACRE, 2005). Possui uma extensão de $30.004,13$ hectares, das quais $2.100,28$ ha $(7 \%)$ integram a zona urbana da cidade de Rio Branco e 27.903,72 ha (93\%) a zona rural dos citados municípios. De sua área localizada na zona rural, $18.148,13$ ha $(65 \%)$ estão desmatados e 11.855,62 ha (35\%) são cobertos por um mosaico de fragmentos florestais primários e secundários em diferentes estádios de regeneração (ACRE, 2005).

O clima local é do tipo quente e úmido com dois períodos climáticos distintos: uma estação chuvosa que se estende de meados de outubro a abril e a estação seca, entre abril e meados de outubro. A precipitação anual varia entre $1773 \mathrm{~mm}$ e $1877 \mathrm{~mm}$ e a temperatura média anual oscila entre $24,5^{\circ} \mathrm{C}$ e $24,7^{\circ} \mathrm{C}$ (ACRE, 2006).

Embora os solos locais predominantes sejam dos tipos Argissolo Amarelo e Argissolo Vermelho, com aptidão para o desenvolvimento de culturas agrícolas, predominam nas áreas desmatadas pastagens usadas na criação extensiva de gado (VIEIRA, 2003). 
Nos remanescentes florestais as tipologias vegetais predominantes são a 'Floresta Aberta com Bambu' e a 'Floresta Aberta com Palmeiras'. Nas poucas manchas de floresta densa são encontradas espécies de interesse comercial como a castanheira (Bertholletia excelsa) e a seringueira (Hevea brasiliensis), embora em densidades inferiores às observadas em outras regiões do Acre (VIEIRA, 2003).

Considerando que as palmeiras apresentam comportamentos diferenciados em ambientes sucessionais distintos (CARVALHO et al., 2010), para o presente estudo foram selecionados três fragmentos florestais secundários com idades estimadas em 5-10 anos, 25-30 anos e 35-40 anos, e um fragmento com floresta primária. Uma breve caracterização destas áreas é apresentada a seguir:

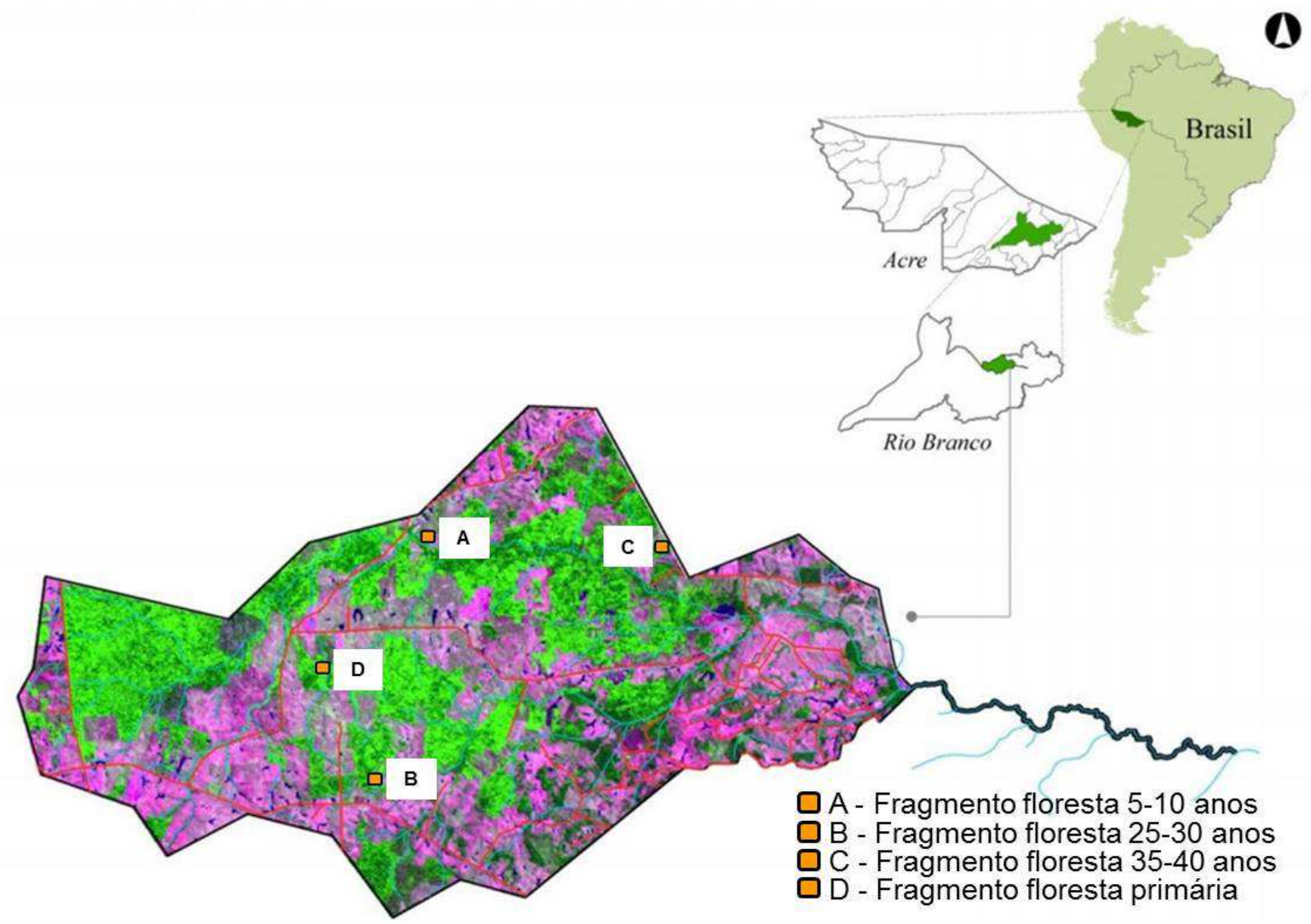

FIGURA 1 - Localização da Área de Proteção Ambiental (APA) do Igarapé São Francisco, Rio Branco, Acre, Brasil, com representação esquemática dos fragmentos florestais avaliados (A-D).

- Fragmento secundário 5-10 anos (área: 145 ha, perímetro: $6.100 \mathrm{~m}$ ): a vegetação local apresenta estrutura vertical irregular e o sub-bosque é dominado pelo bambu (Guadua spp.). O perímetro do fragmento é delimitado, em partes equivalentes, por áreas de pastagens e uma mata ciliar antropizada que margeia um córrego;

- Fragmento secundário 25-30 anos (área: 258 ha, perímetro: $11.100 \mathrm{~m}$ ): a estrutura da vegetação também é bastante irregular, mas a presença do bambu (Guadua spp.) não é tão intensa. O dossel é mais denso e são observados indivíduos de espécies pioneiras (Cecropia spp.) com porte avantajado;

- Fragmento secundário 35-40 anos (área: 51 ha, perímetro: $2.900 \mathrm{~m}$ ): a maioria dos indivíduos arbóreos é de grande porte, porém o dossel ainda é esparso 
e permite a entrada excessiva de luz até o solo florestal. Existem áreas de agricultura familiar com predomínio de plantios de hortaliças e pastagem no entorno do fragmento;

- Fragmento de floresta primária (área: 181 ha, perímetro: $8.400 \mathrm{~m}$ ): As árvores possuem grande porte e o dossel é típico de florestas climácicas. O subbosque é diminuto, no entanto foi observada a presença de clareiras onde espécies pioneiras e invasoras estão em processo de desenvolvimento.

\section{Amostragem e coleta de dados}

Em cada fragmento foi instalada, a uma distância mínima de $100 \mathrm{~m}$ da borda, uma parcela medindo $100 \mathrm{~m}$ de comprimento x $20 \mathrm{~m}$ de largura $\left(2.000 \mathrm{~m}^{2}\right)$, subdividida em quatro subparcelas de $25 \mathrm{~m} \mathrm{X} 20 \mathrm{~m}\left(500 \mathrm{~m}^{2}\right)$ para facilitar a coleta dos dados (PINHEIRO et al., 2015).

Todas as palmeiras encontradas nas parcelas foram contabilizadas e identificadas. A identificação botânica foi realizada in loco pelo Dr. Evandro Ferreira (especialista na família), no herbário UFACPZ e com o uso de bibliografia especializada (LORENZI et al., 2010). Para medir a altura das plantas foi usada trena métrica nos indivíduos com até $1,5 \mathrm{~m}$. Acima disso a altura foi estimada. Indivíduos com estipes cespitosos foram contabilizados unitariamente e as medidas de altura foram tomadas com base no estipe mais desenvolvido.

$\mathrm{Na}$ avaliação da estrutura etária dos indivíduos, foi adotada a seguinte classificação, com base em quatro classes de tamanho:

Classe 1: indivíduos com até $50 \mathrm{~cm}$ de altura;

Classe 2: indivíduos sem estipe aparente, mais de $50 \mathrm{~cm}$ e até $1 \mathrm{~m}$ altura;

Classe 3: indivíduos sem estipe aparente, com mais de $1 \mathrm{~m}$ de altura;

Classe 4: indivíduos com estipe aparente, com mais de $1 \mathrm{~m}$ de altura.

\section{Avaliação florística e fitossociológica}

A análise fitossociológica envolveu as estimativas dos parâmetros da estrutura horizontal da comunidade, de modo a conhecer a importância de cada espécie. Para cada espécie foram calculados os seguintes parâmetros: densidade absoluta e relativa, frequência absoluta e relativa.

\section{a) Densidade}

A densidade, também chamada abundância, é o número de indivíduos de cada espécie na composição do povoamento. O estimador da densidade absoluta $\left(D A_{i}\right)$ e relativa $\left(D R_{i}\right)$ da i-ésima espécie, é obtido pelas seguintes expressões:

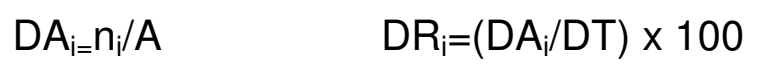

Onde:

$\mathrm{DA}_{\mathrm{i}}=$ densidade absoluta da i-ésima espécie (no.de indivíduos por hectare);

$\mathrm{n}_{\mathrm{i}}=$ número de indivíduos da i-ésima espécie na amostragem;

$\mathrm{N}$ = número total de indivíduos amostrados;

$A$ = área total amostrada, em hectare;

$\mathrm{DR}_{\mathrm{i}}=$ densidade relativa (\%) da i-ésima espécie;

DT $=$ densidade total (soma das densidades de todas as espécies).

Maiores valores de $D A_{i}$ e $D R_{i}$ indicam a existência de um maior número de indivíduos por hectare da espécie no povoamento amostrado. 
b) Frequência

A frequência está relacionada à presença ou ausência de uma espécie nas parcelas amostrais, sem importar o número de indivíduos com que ocorre. Ela é expressa em termos percentuais e indica a dispersão de uma espécie na área amostrada. A freqüência absoluta $\left(F A_{i}\right)$ indica o número de vezes que uma dada espécie ocorre nas parcelas amostrais e a freqüência relativa $\left(F R_{i}\right)$ indica a frequência de uma espécie, relativamente às outras espécies da comunidade amostrada. Maiores valores de $F A_{i}$ e $F R_{i}$ indicam que a espécie está bem distribuída horizontalmente ao longo do povoamento amostrado.

O estimador da frequência absoluta $\left(F A_{i}\right)$ e relativa $\left(F R_{i}\right)$ da i-ésima espécie é obtido pelas seguintes expressões:

$$
\mathrm{FA}_{\mathrm{i}}=\left(\frac{u \mathrm{i}}{u \mathrm{i}}\right) \times 100 \quad \mathrm{FR}_{\mathrm{i}}=\left(\frac{F^{A \mathrm{i}}}{\sum_{\mathrm{l}=\mathrm{i}} F_{\mathrm{ii}}}\right) \times 100
$$

Onde:

$F A_{i}=$ frequência absoluta da i-ésima espécie na comunidade vegetal;

$\mathrm{FR}_{\mathrm{i}}=$ frequência relativa da i-ésima espécie na comunidade vegetal;

$\mathrm{u}_{\mathrm{i}}=$ número de unidades amostrais em que a i-ésima espécie ocorre;

$\mathrm{u}_{\mathrm{t}}=$ número total de unidades amostrais;

$\mathrm{P}=$ número de espécies amostradas.

Os dados foram tabulados e os parâmetros fitossociológicos foram calculados e representados graficamente utilizando planilhas do Microsoft Excel, conforme procedimento adotado por OLIVEIRA et al. (2015).

\section{RESULTADOS E DISCUSSÃO}

\section{Composição florística}

As coletas de dados resultaram em 427 indivíduos em diferentes estágios de crescimento, pertencentes a 11 gêneros e 16 espécies de palmeiras nas quatro áreas estudadas (Tabela 1). SILVA et al. (2014) encontraram 12 gêneros e 16 espécies de palmeiras em áreas de florestas com e sem bambu dominante no subbosque na APA Igarapé São Francisco. Na APA Raimundo Irineu Serra, nas cercanias de Rio Branco, CARVALHO et al. (2010) identificaram 12 gêneros e 19 espécies de palmeiras em fragmentos florestais primários e secundários.

Considerando os resultados obtidos por Ferreira (1998) em seu estudo da flora de palmeiras do 'Parque Natural do Seringueiro' (45 hectares), no município de Plácido de Castro, Acre, onde foram encontrados 10 gêneros e 22 espécies de palmeiras, a flora de palmeiras nos fragmentos florestais avaliados na APA 'Igarapé São Francisco' (635 ha) é pobre, com apenas 11 gêneros e 16 espécies.

Os gêneros mais abundantes foram Bactris (3 spp.) e Attalea, Oenocarpus e Geonoma (2 spp., cada). Os gêneros Aiphanes, Astrocaryum, Desmoncus, Euterpe, Iriartea, Phytelephas, Socratea apresentaram apenas uma espécie (Tabela 1). As espécies Aiphanes aculeata, Geonoma deversa, Iriartea deltoidea, Oenocarpus bataua e Socratea exorrhiza ocorreram apenas em floresta primária, enquanto Astrocaryum ulei foi a única presente em todas as áreas (Tabela 1). As espécies mais abundantes foram Attalea phalerata $(n=185)$, Phytelephas macrocarpa $(n=57)$ e 
Astrocaryum ulei $(\mathrm{n}=52)$, que juntam representaram $68,8 \%$ de todos os indivíduos amostrados no levantamento (Tabela 1).

Em área florestal sem bambu dominante na APA Igarapé São Francisco, SILVA et al. (2014) encontraram que as espécies mais abundantes foram, respectivamente, Astrocaryum ulei $(n=125)$, Attalea phalerata $(n=112)$ e Attalea butyracea $(n=108)$. Juntas, essas três espécies representam $64,85 \%$ dos indivíduos amostrados nesta tipologia. CARVALHO et al. (2010), encontraram em fragmentos primários e secundários (em diferentes estádios sucessionais) que as espécies mais abundantes foram Attalea phalerata, Astrocaryum ulei, Phytelephas macrocarpa e Bactris maraja. Eles verificaram ainda que o fato de a espécie $A$. phalerata ter sido a mais abundante em todos os fragmentos avaliados sugere que a recomposição das comunidades de palmeiras na área era anormal tendo em vista que esta espécie é mais frequente em estádios iniciais de regeneração de florestas secundárias.

TABELA 1 - Número de indivíduos por espécie de palmeiras encontradas nas parcelas de florestas secundárias e de floresta primária, na Área de Proteção Ambiental 'Igarapé São Francisco' em Rio Branco, Acre, Brasil.

\begin{tabular}{|c|c|c|c|c|c|}
\hline \multirow[b]{2}{*}{ NOME CIENTÍFICO } & \multicolumn{4}{|c|}{ PARCELAS } & \multirow[b]{2}{*}{ TOTAL } \\
\hline & $\begin{array}{c}5-10 \\
\text { ANOS }\end{array}$ & $\begin{array}{l}25-30 \\
\text { ANOS }\end{array}$ & $\begin{array}{l}35-40 \\
\text { ANOS }\end{array}$ & PRIMÁRIA & \\
\hline $\begin{array}{l}\text { Attalea phalerata Mart. ex } \\
\text { Sprengel }\end{array}$ & 0 & 20 & 165 & 0 & 185 \\
\hline $\begin{array}{l}\text { Phytelephas macrocarpa Ruiz \& } \\
\text { Pav }\end{array}$ & 0 & 4 & 53 & 0 & 57 \\
\hline Astrocaryum ulei Burret & 12 & 13 & 25 & 2 & 52 \\
\hline Euterpe precatoria Martius & 0 & 1 & 0 & 23 & 24 \\
\hline Bactris concinna Martius & 5 & 0 & 14 & 2 & 21 \\
\hline Oenocarpus mapora H. Karst. & 1 & 5 & 0 & 14 & 20 \\
\hline Oenocarpus bataua Mart. & 0 & 0 & 0 & 19 & 19 \\
\hline Geonoma acaulis Martius & 0 & 1 & 4 & 10 & 15 \\
\hline $\begin{array}{l}\text { Attalea butyracea (Mutis ex L.f.) } \\
\text { Wess. Boer }\end{array}$ & 0 & 0 & 3 & 6 & 9 \\
\hline Bactris maraja Mart. & 2 & 2 & 3 & 0 & 7 \\
\hline Desmoncus mitis Martius & 1 & 2 & 2 & 0 & 5 \\
\hline Bactris dahlgreniana Glassman & 0 & 3 & 0 & 1 & 4 \\
\hline Iriartea deltoidea Ruiz \& Pav & 0 & 0 & 0 & 4 & 4 \\
\hline Geonoma deversa (Point.) Kunth & 0 & 0 & 0 & 3 & 3 \\
\hline Aiphanes aculeata Willd & 0 & 0 & 0 & 1 & 1 \\
\hline $\begin{array}{l}\text { Socratea exorrhiza (Mart) H. } \\
\text { Wendl }\end{array}$ & 0 & 0 & 0 & 1 & 1 \\
\hline TOTAL & 21 & 51 & 269 & 86 & 427 \\
\hline
\end{tabular}

O fragmento florestal com 5-10 anos, cujo sub-bosque era dominado por bambu (Guadua spp.) foi o que apresentou o menor número de espécies (5). Nenhuma delas era tolerante à sombra e a maioria $(B$. concinna, $B$. maraja e $D$. mitis) de pequeno porte e espinhosas. As outras espécies ( $A$. ulei e $O$. mapora) são espécies de médio porte. SILVA et al. (2014) também observaram a ocorrência dessas em área de floresta com bambu dominante. 


\section{Estrutura fitossociológica}

A espécie $A$. phalerata foi a mais abundante dentre todas as espécies encontradas ( $D R=61,34 \%$ ), mas não foi encontrada no fragmento secundário com 510 anos e na floresta primária ( $F R=18,18 \%$ ) (Tabela 2). A maior densidade dessa espécie ocorreu no fragmento secundário com 35-40 anos, onde foram encontrados $165(89,1 \%)$ dos 185 indivíduos avaliados. Esse resultado contraria a afirmação de CARVALHO et al. (2010) que sugeriram que esta espécie é dominante em áreas secundárias recentes.

A presença de $A$. ulei em todos os fragmentos era esperada e confirmam os resultados de CARVALHO et al. (2010) e de SILVA et al. (2014). No presente estudo os resultados de densidade relativa (DR) mostram que esta é uma espécie mais abundante em áreas florestais em início de sucessão (Tabela 2).

A maior densidade relativa das espécies $E$. precatoria $(\mathrm{DR}=26,74 \%)$ e de $O$. bataua $(\mathrm{DR}=22,06 \%)$ no fragmento de floresta primária confirma o caráter climácico das mesmas, que são tolerantes à sombra típica do interior de florestas maduras, onde conseguem germinar e chegar à fase adulta. Este mesmo fragmento foi também o que apresentou o maior número de espécies (12 das 16 identificadas, Tabela 1) e a presença de $I$. deltoidea e $S$. exorrhiza, além das duas espécies citadas acima, indica que o mesmo apresenta bom estado de conservação.

\section{Estrutura etária}

Nenhum dos fragmentos avaliados apresentou populações com uma distribuição de classes de tamanho seguindo o padrão-J invertido ou exponencial negativa na qual se verifica uma predominância de indivíduos jovens (de menor porte) e um decréscimo nas classes superiores, condição interpretada como indicadora de regeneração da floresta que tanto pode resultar na estabilidade como no incremento populacional (BAMBOLIM \& WOJCIECHOWSKI, 2017).

Verificou-se que no fragmento com idade entre 5-10 anos as plântulas estavam ausentes (classe 1) e nas demais seu número era baixo (Figura 2A-D). A ausência de plântulas no fragmento com 5-10 anos pode ser resultado da ocorrência de queimadas recentes que atingiram o sub-bosque ou pelo fato do mesmo ser dominado por bambu (Guadua spp.) que, quando dominante, tende a suprimir fisicamente a regeneração de outras espécies (GRISCOM \& ASHTON, 2003).

Este fragmento foi também o que apresentou o menor número de indivíduos (Tabela 1) e essa condição confirma a sugestão de SCARIOT (2000) de que o número de palmeiras em uma área florestal é muito influenciado pela quantidade de plântulas que, por sua vez, é o estágio de vida mais afetado pelo processo de fragmentação florestal. Já SILVA et al. (2014) encontraram maior número de indivíduos nas classes iniciais (1,2 e 3) em fragmentos de floresta com e sem bambu (Guadua spp.) na APA Igarapé São Francisco, no Acre, no entanto chegaram à conclusão que a estrutura populacional de palmeiras encontra-se estável e em processo de autoregeneração somente na área sem bambu.

PINHEIRO et al. (2015) também identificaram maior número de indivíduos nas classes iniciais em diferentes gradientes topográficos presentes na Reserva Florestal Humaitá, Acre e para os demais fragmentos avaliados neste estudo, a baixa quantidade de plântulas encontradas pode ser resultante do pequeno número de indivíduos reprodutivos encontrados ou do isolamento desses fragmentos de áreas matrizes do entorno que poderiam servir como fonte de sementes (CARVALHO et al., 2010). 
TABELA 2 - Parâmetros fitossociológicos das espécies de palmeiras encontradas nos fragmentos de floresta secundária e primária da Área de Proteção Ambiental 'Igarapé São Francisco' em Rio Branco, Acre, Brasil. DR = Densidade relativa $(\%) ; F R=$ frequência relativa $(\%)$.

\begin{tabular}{|c|c|c|c|c|c|c|c|c|}
\hline \multirow{3}{*}{ Espécies } & \multicolumn{8}{|c|}{ Fragmentos } \\
\hline & \multicolumn{2}{|c|}{$5-10$ anos } & \multicolumn{2}{|c|}{ 25-30 anos } & \multicolumn{2}{|c|}{$35-40$ anos } & \multicolumn{2}{|c|}{ Primária } \\
\hline & FR & DR & FR & DR & FR & DR & FR & DR \\
\hline Aiphanes aculeata Willd & - & - & - & - & - & - & 4,17 & 1,16 \\
\hline Astrocaryum ulei Burret & 25 & 57,14 & 8,33 & 25,49 & 9,09 & 9,29 & 4,17 & 2,33 \\
\hline Attalea butyracea (Mutis ex L.f.) Wess. Boer & - & - & - & - & 13,64 & 1,12 & 8,33 & 6,98 \\
\hline Attalea phalerata Mart. ex Sprengel & - & - & 16,67 & 39,22 & 18,18 & 61,34 & - & - \\
\hline Bactris concinna Martius & 18,8 & 23,81 & - & - & 13,64 & 5,2 & 4,17 & 2,33 \\
\hline Bactris dahlgreniana Glassman & - & - & 12,5 & 5,88 & - & - & 12,5 & 1,16 \\
\hline Bactris maraja Mart. & 18,8 & 9,52 & 8,33 & 3,92 & 13,64 & 1,12 & - & - \\
\hline Desmoncus mitis Martius & 18,8 & 4,76 & 12,5 & 3,92 & 9,09 & 0,74 & - & - \\
\hline Euterpe precatoria Martius & - & - & 12,5 & 1,96 & - & - & 4,17 & 26,74 \\
\hline Geonoma acaulis Martius & - & - & 8,33 & 1,96 & 13,64 & 1,49 & 12,5 & 11,63 \\
\hline Geonoma deversa (Point.) Kunth & - & - & - & - & - & - & 12,5 & 3,49 \\
\hline Iriartea deltoidea Ruiz \& Pav & - & - & - & - & - & - & 8,33 & 4,65 \\
\hline Oenocarpus bataua Mart. & - & - & 8,33 & 9,8 & - & - & 16,67 & 22,09 \\
\hline Oenocarpus mapora H. Karst. & 18,8 & 4,76 & - & - & - & - & 8,33 & 16,28 \\
\hline Phytelephas macrocarpa Ruiz \& Pav & - & - & 12,5 & 7,84 & 9,09 & 19,7 & - & - \\
\hline Socratea exorrhiza (Mart) H. Wendl & - & - & - & - & - & - & 4,17 & 1,16 \\
\hline
\end{tabular}



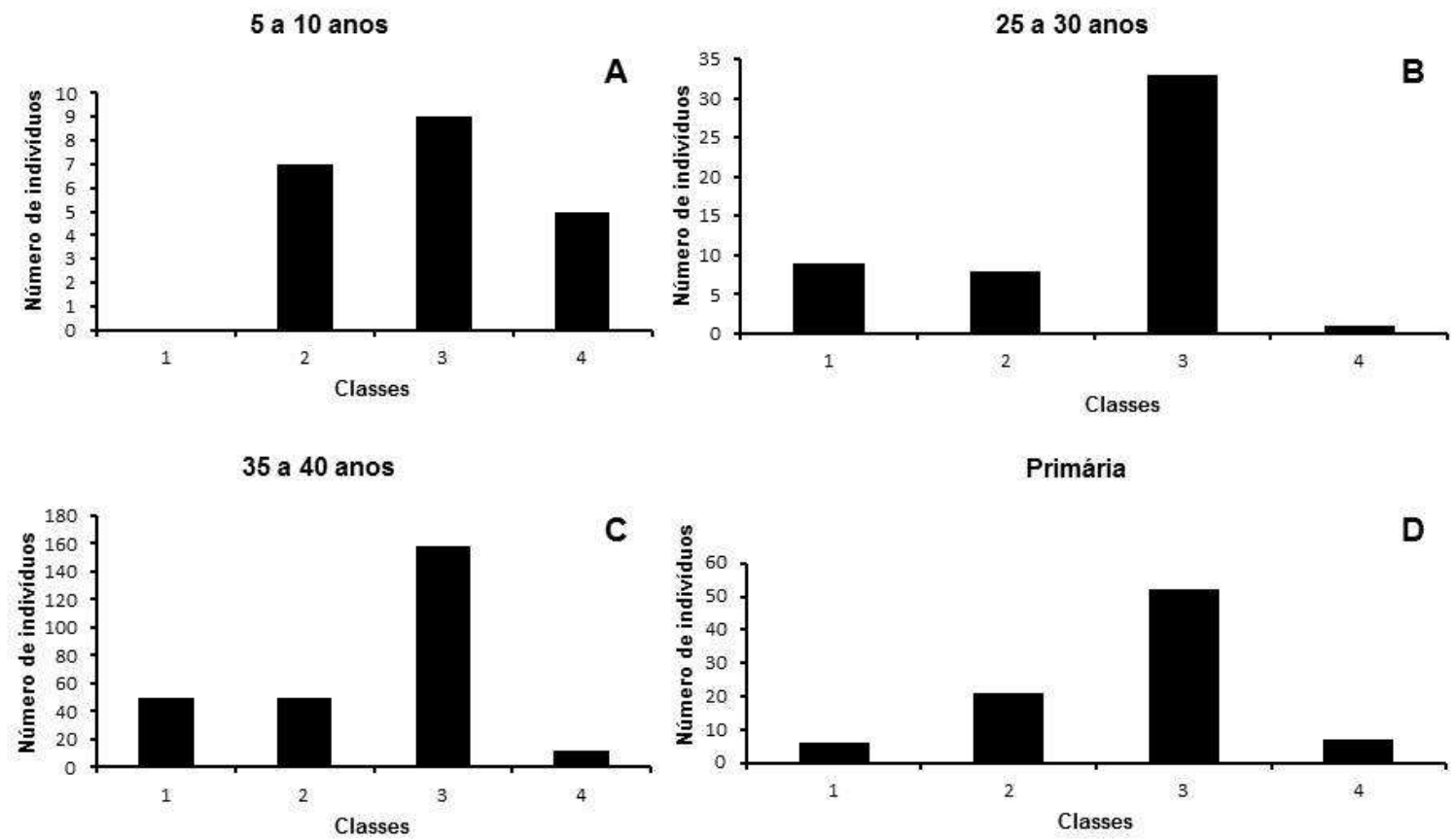

FIGURA 2 - Estrutura etária dos indivíduos de palmeiras encontrados em levantamento realizado na Área de Proteção Ambiental (APA) do Igarapé São Francisco, Rio Branco, Acre, Brasil. A - Fragmento florestal com 5-10 anos; B - Fragmento florestal com 25-30 anos; C Fragmento florestal com 35-40 anos; D - Fragmento florestal primário.

Em todos os fragmentos pode-se observar que a classe de indivíduos mais numerosa foi a 3. A grande abundância das espécies de médio a grande porte $A$. phalerata, A. ulei, E. precatoria e $O$. bataua nas parcelas avaliadas explica em parte essa situação porque indivíduos dessas espécies com mais de $1 \mathrm{~m}$ de altura e sem estipe aparente são menos suscetíveis à predação por exibirem grande número de folhas com grande porte. A. ulei foi observada como a espécie mais abundante no estudo realizado por SILVA et al. (2013) em uma área de encosta na Floresta Estadual do Rio Gregório enquanto $E$. precatoria e $O$. bataua também foram observadas por GOMES et al. (2016) em estudo realizado em florestas primárias localizadas no sul do estado de Roraima.

\section{CONCLUSÕES}

- A flora de palmeiras encontrada nos fragmentos florestais avaliados durante o presente estudo é pobre se comparada com os resultados de outros estudos realizados na região;

- Quanto maior a idade do fragmento, maior é a riqueza de gêneros e espécies;

- Em todos os fragmentos avaliados, as comunidades de palmeiras apresentam estrutura etária anormal, com destaque para a ausência ou o baixo número de plântulas;

- A espécie mais abundante foi Attalea phalerata, reconhecida como típica de florestas em estágios iniciais de regeneração, mas que foi dominante no 
fragmento com 35-40 anos, sugerindo que as alterações antrópicas e a fragmentação da paisagem na APA podem estar favorecendo essa espécie;

- No fragmento com idade mais recente (5-10 anos) a existência da comunidade de palmeiras parece estar comprometida por alterações antrópicas (incêndios florestais recorrentes), por efeitos da fragmentação e pela invasão e dominância do bambu (Guadua spp.) no sub-bosque do fragmento.

\section{REFERÊNCIAS}

ACRE. Governo do Estado do Acre. Programa Estadual de Zoneamento Ecológico Econômico do Estado do Acre. Zoneamento ecológico-econômico do Acre fase II: documento síntese - escala 1:250.000. Rio Branco: SEMA, 2006, 356p.

ACRE. Governo do Estado do Acre. Secretaria de Estado de Meio Ambiente e Recursos Naturais - SEMA. Peça de criação da Área de Proteção Ambiental (APA) Igarapé São Francisco: Unnidade de conservação de uso sustentável. Rio Branco: SEMA, 2005, 35p.

ARAÚJO, L. H. B.; SILVA, R. A. R.; CHAGAS, K. P. T.; NÓBREGA, C. C.; SANTANA, J. A. S. Composição florística e estrutura fitossociológica de um fragmento de Floresta Ombrófila Densa no município de Macaíba, RN. Revista Agro@mbiente, v. 9, n. 4, p. 455-464, 2015. Disponível em: <http://dx.doi.org/10.18227/1982-8470ragro.v9i4.2441>. DOI:10.18227/19828470ragro.v9i4.2441.

ARIMA, E. Y.; WALKER, R. T.; PERZ, S.; SOUZA-JUNIOR, C. Explaining the fragmentation in the Brazilian Amazonian Forest. Journal of Land Use Science, v. 11, n. 3, p.257-277, 2016. Disponível em: < http://dx.doi.org/10.1080/1747423X.2015.1027797>.

DOI:10.1080/1747423X.2015.1027797.

ARROYO-RODRÍGUEZ, V.; RÖS, M.; ESCOBAR, F.; MELO, F. P. L.; SANTOS, B. A.; TABARELLI, M.; CHAZDON, R. Plant $\beta$-diversity in fragmented rain forests: testing floristic homogenization and differentiation hypotheses. Journal of Ecology, v. 101, n. 6, p. 1449-1458, 2013. Disponível em: <http://dx.doi.org/10.1111/1365-2745.12153>. DOI:10.1111/1365-2745.12153.

BAMBOLIM, A.; WOJCIECHOWSKI, J. C. Composição florística e fitossociológica de um remanescente de floresta ombrófila mista. Revista de Agricultura Neotropical, v. $4, \quad$ n. $1, \quad$ p. 28-35, 2017. Disponível em: <http://periodicosonline.uems.br/index.php/agrineo/article/view/1173>. Acesso em: 06 mar 2017.

BENCHIMOL, M.; PERES, C. A. Edge-mediated compositional and functional decay of tree assemblages in Amazonian forest islands after 26 years of isolation. Journal of Ecology, v. 103, n. 2, p. 408-420, 2015. Disponível em: <http://dx.doi.org/10.1111/1365-2745.12371>. DOI:10.1111/1365-2745.12371.

CARVALHO, A. L.; FERREIRA, E. J. L.; LIMA, J. M. T. Comparações florísticas e estruturais entre comunidades de palmeiras em fragmentos de floresta primária e 
secundária da Área de Proteção Ambiental Raimundo Irineu Serra - Rio Branco, Acre, Brasil. Acta Amazônica, v. 40, n. 4, p.657-666, 2010. Disponível em: <http://dx.doi.org/10.1590/S0044-59672010000400004>. DOI: 10.1590/S004459672010000400004.

CHAVES, A. D. C. G.; SANTOS, R. M. S.; SANTOS, J. O.; FERNANDES, A. A.; MARACAJÁ, P. B. A importância dos levantamentos florístico e fitossociológico para a conservação e preservação das florestas. Revista ACSA, v. 9, n. 2, p. 42-48, $2013 . \quad$ Disponível em: <http://revistas.ufcg.edu.br/acsa/index.php/ACSA/article/view/449/pdf>. Acesso em: 06 mar 2017.

FERREIRA, E. J. L. Palmeiras do Parque Natural do Seringueiro, Acre, Brasil. Acta Amazonica, v. 28, n. 4, p. 373-394, 1998. Disponível em: <http://dx.doi.org/10.1590/1809-43921998284394>. DOI: 10.1590/180943921998284394.

GOMES J. P.; CONDE, T. M.; SANTOS, R. de L.; DIONISIO, L. F. S.; DUARTE, O. R.; MIRANDA, D. L. D. de; SILVA, F. da. Efeitos de gradientes ambientais na fitossociologia de assembleias de palmeiras no sudeste de Roraima, Brasil. Nativa, v. 4, n. 5, p. 317-327, 2016. Disponível em: < http://dx.doi.org/10.14583/23187670.v04n05a08>. DOI: 10.14583/2318-7670.v04n05a08

GRISCOM, B. W.; ASHTON, P. M. S. Bamboo control of forest succession: Guadua sarcocarpa in southeastern Peru. Forest Ecology and Management, v. 175, n. 1-3, p. 445-454, 2003. Disponível em: <http://dx.doi.org/10.1016/S0378-1127(02)002141>. DOI: 10.1016/S0378-1127(02)00214-1.

LAURANCE, W. F.; VASCONCELOS, H. L. Consequências ecológicas da fragmentação florestal na Amazônia. Oecologia Brasiliensis, v. 13, n. 3, p. 434451, 2009. Disponível em: <http://dx.doi.org/10.4257/oeco.2009.1303.03>. DOI:10.4257/oeco.2009.1303.03.

LORENZI, H.; NOBLICK, L.; KAHN, F.; FERREIRA, E. J. L. Flora Brasileira: Arecaceae (Palmeiras). Nova Odessa: Plantarum, 2010. 384p.

MEDEIROS, H.; CASTRO, W.; SALIMON, C. I; SILVA, I. B.; SILVEIRA, M. Tree mortality, recruitment and growth in a bamboo dominated forest fragment in southwestern Amazonia, Brazil. Biota Neotropica, v. 13, n. 2, p. 29-34, 2013. Disponível em: <http://www.biotaneotropica.org.br/v13n2/pt/fullpaper?bn00613022013+en>. Acesso em: 06 mar 2017.

MESQUITA, R. C. G.; MASSOCA, P. E. S.; JAKOVAC, C. C.; BENTOS, T. V.; WILLIAMSON, G. B. Amazon rain forest succession: stochasticity or land-use legacy? Bioscience, v.65, n.9, p.849-861, 2015. Disponível em: <https://doi.org/10.1093/biosci/biv108>. DOI:10.1093/biosci/biv108.

OLIVEIRA, E. K. B.; NAGY, A. C. G.; BARROS, Q. S.; MARTINS, B. C.; MURTAJÚNIOR, L. S. Composição florística e fitossociológica de fragmento florestal no 
sudoeste da Amazônia. Enciclopédia Biosfera, v. 11, n. 21, p. 2126-2146, 2015. Disponível em: $<$ http://www.conhecer.org.br/enciclop/2015b/biologicas/composicao\%20floristica.pdf >. Acesso em: 06 mar 2017.

PINHEIRO, R. M.; FERREIRA, E. J. L.; SANTOS, E. A. Florística e fitossociologia de comunidades de palmeiras na Reserva Florestal Humaitá, Acre. Enciclopédia Biosfera, v. 11, n. 22, p. 276-292, 2015. Disponível em: <http://dx.doi.org/10.18677/Enciclopedia_Biosfera_2015_086>.

DOI:10.18677/Enciclopedia_Biosfera_2015_086.

PÜTZ, S.; GROENEVELD, J.; HENLE, K.; KNOGGE, C.; MARTENSEN, A.C.; METZ, M.; METZGER, J. P.; RIBEIRO, M. C.; PAULA, M. D.; HUTH, A. Long-term carbon loss in fragmented Neotropical forests. Nature Communications, v. 5, p. 5037, 2014. Disponível em: <http://dx.doi.org/10.1038/NCOMMS6037>. DOI:10.1038/ncomms6037.

SANTO-SILVA, E. E.; ALMEIDA, W. R.; MELO, F. P.; ZICKEL, C. S.; TABARELLI, $M$. The nature of seedling assemblages in a fragmented tropical landscape: implications for forest regeneration. Biotropica, v. 45, n. 3, p. 386-394, 2013. Disponível em: <http://dx.doi.org/10.1111/btp.12013>. DOI:10.1111/btp.12013.

SCARIOT, A. O. Seedling mortality by litterfall in Amazonian forest fragments. Biotropica, v. 32, n. 4, p. 662-669, 2000. Disponível em: <http://dx.doi.org/ 10.1111/j.1744-7429.2000.tb00513.x>. DOI:10.1111/j.1744-7429.2000.tb00513.x.

SCARIOT, A. O. Forest fragmentation effects on palm diversity in central Amazonia. Journal of ecology, v. 87, n. 1, p. 66-76, 1999. Disponível em: <http://dx.doi.org/ 10.1046/j.1365-2745.1999.00332.x>. DOI:10.1046/j.1365-2745.1999.00332.x.

SILVA, A. C.; HIGUCHI, P.; NEGRINI, M.; GRUDTNER, A.; ZECH, D. F. Caracterização fitossociológica e fitogeográfica de um trecho de floresta ciliar em Alfredo Wagner, SC, como subsídio para restauração ecológica. Ciência Florestal, v. 23, n. 4, p. 579-593, 2013. Disponível em: <http://dx.doi.org/10.5902/1980509812342>. DOI: 10.5902/1980509812342.

SILVA, F.; SUWA, R.; KAJIMOTO, T.; ISHIZUKA, M.; HIGUCHI, N.; KUNERT, N. Allometric equations for estimating biomass of Euterpe precatoria, the most abundant palm species in the Amazon. Forests, v. 6, n. 2, p. 450-463, 2015. Disponível em: <http://dx.doi.org/ 10.3390/f6020450>. DOI:10.3390/f6020450.

SILVA, G. M.; LIMA, A. F.; MENDONÇA, C. C.; SILVA, A. S.; FERREIRA, E. J. L. Aspectos florísticos e fitossociológicos de comunidades de palmeiras (Arecaceae) em fragmentos florestais com e sem bambu (Guadua spp.) na APA Igarapé São Francisco, Acre. Enciclopédia Biosfera, v. 10, n. 18, p. 403-426, 2014. Disponível em:

<http://www.conhecer.org.br/enciclop/2014a/AGRARIAS/aspectos\%20floristicos.pdf> . Acesso em: 06 mar 2017. 
TABARELLI, M.; AGUIAR, A. V.; RIBEIRO, M. C.; METZGER, J. P. A conversão da floresta atlântica em paisagens antrópicas: lições para a conservação da diversidade biológica das florestas tropicais. Interciencia, Caracas, v. 37, n. 2, pp. 88-92, 2012. Disponível em: <http://www.redalyc.org/pdf/339/33922717002.pdf>. Acesso em: 06 mar 2017.

ter STEEGE, H.; PITMAN, N. C. A.; SABATIER, D.; BARALOTO, C.; SALOMÃO, R. P.; GUEVARA, J. E. et al. Hyperdominance in the Amazonian Tree Flora. Science, v. $342, \quad$ n. 6156, 1243092, 2013. Disponível em: <http://dx.doi.org/10.1126/science.1243092>. DOI:10.1126/science.1243092.

TILMAN, D.; MAY, R.M.; LEHMAN, C.L.; NOWAK, M.A. Habitat destruction and the extinction debt. Nature, v. 371, p. 65-66, 1994. Disponível em: <http://dx.doi.org/ 10.1038/371065a0>. DOI: 10.1038/371065a0.

VIEIRA, L. J. S. 2003. Diagnóstico ambiental e socioeconômico da bacia hidrográfica do Igarapé São Francisco. Rio Branco: Prefeitura Municipal de Rio Branco. 100p. 